\title{
Diacronie
}

Studi di Storia Contemporanea

$N^{\circ} 27,3$ | 2016

Stato, costituzione e democrazia

\section{Nota introduttiva n. 27 - settembre 2016}

Jacopo Bassi e Fausto Pietrancosta

\section{(2) OpenEdition}

\section{Journals}

\section{Edizione digitale}

URL: http://journals.openedition.org/diacronie/4193

DOI: $10.4000 /$ diacronie.4193

ISSN: 2038-0925

\section{Editore}

Association culturelle Diacronie

\section{Notizia bibliografica digitale}

Jacopo Bassi e Fausto Pietrancosta, « Nota introduttiva n. 27 - settembre 2016 », Diacronie [Online], $N^{\circ} 27,3$ | 2016, Messo online il 29 septembre 2016, consultato il 24 septembre 2020. URL : http:// journals.openedition.org/diacronie/4193; DOI : https://doi.org/10.4000/diacronie.4193

Questo documento è stato generato automaticamente il 24 settembre 2020.

Creative Commons License 


\section{Nota introduttiva n. 27 - settembre 2016}

Jacopo Bassi e Fausto Pietrancosta

Stato, costituzione e democrazia. Confronti, strumenti di navigazione e di diffusione del sapere

I. Progettare il futuro, ripensare lo Stato: diplomazia, eversione, contestazione e violenza 
1 La prima sezione del numero $27 \mathrm{di}$ Diacronie è dedicata agli articoli inediti. In questa occasione presentiamo cinque saggi che, seppur con modalità e approcci molto diversi, ruotano intorno all'idea di Stato.

Il saggio proposto da Roberto Sciarrone, partendo dall'esame del panorama geopolitico internazionale, si interroga sullo "stato" della società ottomana alla vigilia della Prima guerra mondiale, analizzando le diverse influenze europee sull'Impero degli Osmanli. Sciarrone

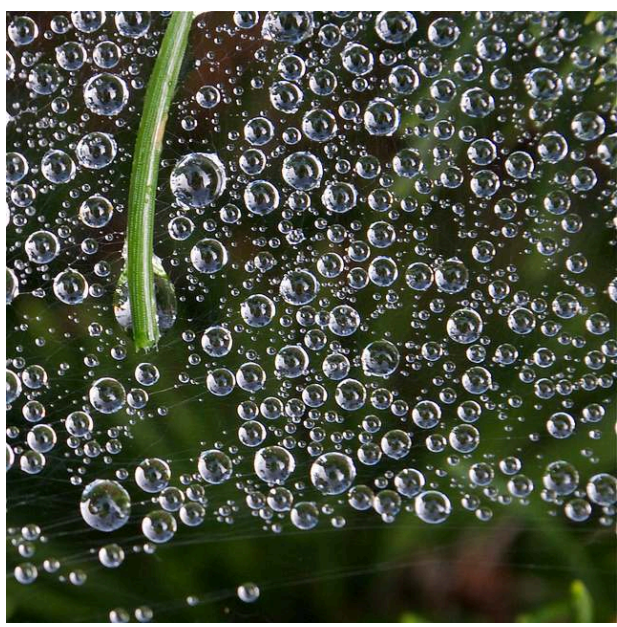
attraverso le carte degli addetti militari italiani in Turchia, custodite presso l'Archivio dell'Ufficio Storico dello Stato Maggiore dell'Esercito, ripercorre la fase terminale della storia ottomana, delineando le nuove prospettive socio-politiche ed economiche foriere del nuovo corso della storia nell'intera area balcanica e vicino orientale.

Lorenzo Spurio Passamonti con il suo articolo prende in analisi i periodici fascisti analizzando le caratteristiche della rivista «La nobiltà della Stirpe». Diretta dal nobile Stefano Mario Cutelli, la rivista promosse una peculiare linea politica che vedeva nella diade monarchia-fascismo la guida ideale per risollevare le sorti dell'Italia. Passamonti, ripercorrendo le vicende e i personaggi di un'avventura editoriale destinata ad andare incontro ad un sostanziale insuccesso, riesce così a rappresentare l'eterogeneità della società fascista e ad indagare uno degli aspetti peculiari del regime: l'utilizzo dello strumento dell'editoria a fini propagandistici.

Differente è invece l'analisi proposta da Nicola Tonietto, il quale indaga in profondità uno dei temi ancora oggi considerati "caldi" nella storia dello Stato italiano. Si tratta del tentativo eversivo - così come lo definisce l'autore - di Junio Valerio Borghese, conosciuto anche con l'appellativo di 'golpe borghese'. Nel saggio in questione si studiano a fondo tutti gli elementi politici, sociali, militari ed anche mafiosi legati al colpo di stato promosso dal "principe nero", ma anche gli effetti prodotti dal suo fallimento: la permanenza dell'Italia nella linea centrista, conservatrice e filo-atlantica promossa, fra gli altri attori politici, anche dagli Stati Uniti.

5 Nel suo articolo Carmine Marino offre un'accurata ricostruzione dei moti di contestazione studentesca che interessarono l'ateneo napoletano nel biennio 1968-1969. Il percorso di indagine sulla documentazione presentata si staglia sullo sfondo dei problemi che affliggevano la città di Napoli, e nel contesto più generale delle tensioni che caratterizzavano il biennio sessantottino a livello nazionale, riuscendo così ad esaltare la vivacità di un ambiente universitario considerato marginale rispetto a quello di altre città e che si rivelerà caparbiamente determinato e propositivo nei contenuti e nelle iniziative, ma drammaticamente inconcludente nella capacità di costruire una proposta politica in grado di coinvolgere e creare il necessario consenso anche al di là dell'ambiente universitario.

Rafaella Bettamio ci riporta sul tema già affrontato nel numero 24 di Diacronie, consacrato alle eredità dei regimi militari. L'autrice si sofferma sul funzionamento dei 
DOI-CODI (Destacamento de Operações de Informação - Centro de Operações de Defesa Interna) messi in opera dalla dittatura militare brasiliana e sull'eredità che hanno lasciato nella prassi operativa delle forze di pubblica sicurezza. Bettamio, facendo riferimento alle osservazioni formulate dalla Commissione per la Verità brasiliana, insiste sulla necessità di fare giustizia; questo non è ancora avvenuto, sottolinea l'autrice, anche per effetto di una Legge di amnistia emanata mentre ancora era al potere il regime militare. Fare giustizia è una necessità per poter riformare e reimpostare su altre basi il modus operandi delle forze di polizia.

\section{Confronti. "Imperfetto perfettismo": proposta per un'analisi comparata del dibattito sulle riforme costituzionali nell'Italia del secondo dopoguerra}

7 Fra le costruzioni e le invenzioni umane, come è noto, molto se non tutto è perfettibile. Nella storia costituzionale italiana questa regola probabilmente vale ancora di più e il confronto su questi temi è sempre stato molto vivace. Partendo da questa premessa la seconda sezione del numero prova a ricostruire, attraverso una serie di interviste curate da Fausto Pietrancosta, un'analisi comparata del dibattito sulle riforme costituzionali e dei tentativi di revisione del testo costituzionale nell'Italia del secondo dopoguerra che hanno determinato la nascita, il consolidamento e l'evoluzione delle istituzioni politiche in età repubblicana.

8 Abbiamo affrontato questo percorso adottando un metodo che ha volutamente rovesciato la prospettiva di analisi cronologica, partendo dall'esame della più recente riforma costituzionale (la cosiddetta "Riforma Boschi")1, approvata in via definitiva dal Parlamento italiano ad inizio del 2016, per poi addentrarci nello studio delle soluzioni adottate e delle modifiche apportate negli anni, fino a risalire alle discussioni e alle scelte con le quali i costituenti, nei mesi di lavoro tra il 1946 e il 1947, hanno contribuito alla redazione degli articoli della seconda parte della Costituzione italiana, quella riguardante l'ordinamento della Repubblica ${ }^{2}$. Lo strumento utilizzato è stato dunque quello dell'intervista, allo scopo di costruire, attraverso poche e mirate domande, sottoposte in modo identico a docenti e studiosi differenti per formazione e approccio ai temi oggetto di studio, un percorso di indagine comparativa delle tappe e dei punti fondamentali del dibattito sulle riforme costituzionali nel secondo dopoguerra.

9 Il confronto a distanza che ne è scaturito è il frutto di questo azzardo metodologico che ci ha permesso di offrire degli originali e variegati strumenti di analisi (giuridici, politologici, storico-filosofici e sociologici) che, oltre ad arricchire il dibattito, sempre vivo e attuale, sui percorsi del costituzionalismo italiano, possono contribuire a orientare la discussione, sia nell'opinione pubblica che fra gli specialisti, verso la formazione di un pensiero sempre più consapevole e supportato da tesi e informazioni chiare e puntuali. Così, se da un lato il quadro complessivo delle interviste presenta nella descrizione degli aspetti specifici del dibattito, e nella valutazione degli elementi tecnici delle riforme, singole visioni e posizioni individuali anche molto diverse, se non opposte, dall'altro lato emerge un quadro di indagine unitario sull'intera vicenda storica dell'Italia repubblicana e delle sue radici nella contemporaneità, che tiene conto dei differenti punti di vista e dei vari ambiti di studio: politico e di conformazione del sistema partitico, di definizione degli organi costituzionali e di funzionamento dei 
meccanismi istituzionali, dei movimenti collettivi, dei risvolti e degli sviluppi sociali, delle implicazioni filosofiche alla base del dibattito sui profili del diritto, e sugli assetti del potere nel corso del Novecento.

10 Nella prima intervista il costituzionalista Salvatore Bonfiglio entra nel vivo del funzionamento del nostro sistema istituzionale evidenziandone le criticità che si sono palesate nel corso degli anni e, attraverso un costante confronto con le formule e le scelte adottate dai membri dell'Assemblea costituente, chiarisce i possibili modi per superarle. In quest'ottica le modifiche apportate dalle più recenti leggi di revisione costituzionale, sino alla riforma approvata ad inizio del 2016, rappresentano gli elementi fondamentali di un percorso di analisi che tiene conto anche delle esperienze istituzionali di altri paesi, e dell'evoluzione del dibattito costituzionale a livello internazionale.

11 Analogamente lo storico Guido Crainz esplora gli aspetti più importanti del dibattito sulla Costituzione italiana che si sono succeduti nel tempo, approfondendo le premesse politiche alla base delle proposte correttive e dei tentativi di modifica, in una prospettiva di comparazione storica delle fasi e delle caratteristiche del percorso di sviluppo delle istituzioni repubblicane. La sua analisi tocca i nodi fondamentali del funzionamento degli organi costituzionali e delle disfunzioni che nel corso dei decenni si sono evidenziate, descrivendo le soluzioni che si sono prospettate, quelle che si sono definite e, in ultimo, quelle adottate con l'ultima riforma approvata.

Lo storico del pensiero politico Carlo Galli partendo dall'esame degli esiti dell'ultima revisione del testo costituzionale, attraverso l'analisi delle ideologie e delle culture politiche in rapporto con le crisi sistemiche del paese, coglie l'occasione per approfondire le differenze tra il processo costituente del 1946-1947 e le successive revisioni del testo costituzionale alla luce del mutato contesto storico. La sua analisi integra in questo modo la descrizione delle dinamiche politiche e istituzionali che hanno caratterizzato la storia politica italiana con la presentazione delle peculiarità del dibattito sulle forme del diritto.

13 Lo storico Giuseppe Carlo Marino, a sua volta, riprende i passaggi fondamentali della storia politica dell'Italia contemporanea ponendo l'accento sugli sviluppi politicoistituzionali legati all'evoluzione degli assetti del potere dall'epoca liberale sino al periodo repubblicano. La sua analisi tende a sviscerare i nodi fondamentali della recente riforma della Costituzione italiana inquadrandola nelle più generali dinamiche politiche, sociali ed economiche globali che hanno provocato quei mutamenti che, ancora oggi, animano il dibattito e condizionano le scelte di quelle classi dirigenti, sempre più inclini ad assecondare gli effetti di quella che suole definire la rivoluzione "elettronico-informatica".

14 Lo storico Paolo Pombeni nella sua intervista ci offre un attento esame della vicenda costituzionale italiana, con il quale, oltre agli sviluppi istituzionali e alle caratteristiche giuridiche e politologiche delle formule, fornisce un importante resoconto delle idee e delle culture politiche alla base dell'evoluzione del dibattito sulla riforma della Costituzione. Attraverso l'analisi dei fattori primari e delle variabili del sistema politico italiano giunge così a ricostruire le principali esperienze e a ripercorrere le tappe fondamentali del percorso di consolidamento e revisione delle istituzioni repubblicane.

Lo storico Valerio Romitelli, infine, presenta le sue valutazioni del dibattito sulle riforme costituzionali nel secondo dopoguerra, dedicando ampio spazio alle dinamiche dal basso che hanno determinato lo svolgersi della vicenda storico-politica del nostro 
paese. I movimenti politici, i corpi intermedi della società e le organizzazioni dell'economia risultano essenziali nella sua analisi, che tiene conto anche degli apporti degli studi filosofico-culturali sulla natura del diritto, e grazie alla quale ci offre una specifica visione dell'evoluzione degli assetti di potere nel percorso storico dell'Italia contemporanea.

16 Sei interviste dunque, sei confronti che pongono l'accento su aspetti differenti, e che mettono in luce punti di vista anche molto diversi, proponendo letture a volte diametralmente opposte della nostra storia e che, lungi dal rappresentare una risposta esaustiva alle domande che ogni appassionato della materia o semplice cittadino potrebbe porsi, rappresentano un contributo per accompagnare il lettore nella ricostruzione e comprensione delle logiche che oggi caratterizzano il funzionamento delle nostre istituzioni, e di quelle che potrebbero animare, nei prossimi anni, quel dibattito politico-costituzionale del nostro paese, sempre teso alla ricerca di un presunto, e inafferrabile, "perfettismo" delle formule giuridiche e dei meccanismi istituzionali che, forse, proprio per sua natura, è imperfetto e intrinsecamente instabile.

\section{Sitografie: Navigare il Mediterraneo e il Baltico sul web}

Due interessanti sitografie (curate da Sante Cruciani e Raffaello Ares Doro e da Deborah Paci) nella terza sezione del numero ci guidano alla scoperta delle istituzioni che si occupano dello studio del Mar Mediterraneo e del Baltico. Una serie di caratteristiche li accomunano: l'essere sostanzialmente chiusi; la dimensione di spazio di apertura e divisione al contempo (nel Baltico fra Est e Ovest, nel Mediterraneo fra Nord e Sud); la stratificazione storica; la pluralità di entità statali che vi si affacciano.

18 Proprio in ragione di questi elementi di complessità, l'analisi di mosaici così ricchi di sfaccettature richiederà approcci plurimi e plurali. Le due sitografie qui proposte offrono un quadro d'insieme che permetterà al lettore di rintracciare alcune piste di ricerca fra le sponde di questi due bacini.

\section{Progetto Interculturale COoperativo (PICO)}

19 La quarta sezione di questo numero è dedicata ad una nuova iniziativa di Diacronie, Progetto Interculturale COoperativo (PICO). Il progetto nasce dalla volontà di mettere a disposizione dei lettori della rivista la produzione di scuole storiografiche di realtà non sempre conosciute nel panorama italiano.

20 Il nome PICO è un omaggio a Pico della Mirandola, ma è anche un acronimo che richiama ad una realtà che vogliamo orizzontale e cooperativa, e orientata ad una diffusione il più possibile capillare della cultura. Spesso la circolazione di idee (e quindi dei saperi) segue percorsi forzati, che portano ad una diffusione delle opere e della conoscenza che finisce per essere egemonizzata da poche lingue (e, conseguentemente, da un numero esiguo di mercati editoriali); in questo contesto le storiografie "minori" scontano, fra loro, una sorta di incomunicabilità: l'unica possibilità per entrare in contatto vicendevolmente è attraverso la mediazione di una lingua terza, in grado di garantire - oltre che la reciproca comprensione - una sorta di verifica dell'appetibilità del pensiero testata su un mercato che si pretende globale. Questa logica ha fatto sì che le opere provenienti dalle periferie storiografiche - ammesso che si possa definirle in 
questi termini - necessitassero di passare da un centro editoriale, grossomodo coincidente con il mondo anglofono: un autore che approdi al mercato globale (anglofono) e ottenga successo ha la possibilità, a quel punto, di vedersi tradotto in una lingua "periferica". Si sottraggono parzialmente a questo sistema le opere in francese, che seppur in forma residuale, in ambito scientifico mantiene ancora il ruolo di lingua internazionale, e quelle in spagnolo che, grazie all'alto numero di ispanofoni, può costituire un mercato a sé.

21 I problemi insiti in un simile meccanismo di diffusione del sapere sono diversi: anzitutto verranno veicolate solo le conoscenze ritenute utili o suscettibili di poter risultare interessanti secondo la prospettiva delle élites che controllano il mercato editoriale globale (potrebbero godere quindi di minor considerazione quelle opere storiograficamente, politicamente o ideologicamente non in linea con il pensiero dominante); in secondo luogo le periferie non potranno condividere fra loro uno scambio di informazioni diretto (con tutto ciò che ne consegue); infine la traduzione stessa dei termini e delle idee passerà attraverso una lingua terza, che fungerà necessariamente da primo vettore.

L'idea alla base di PICO è proprio quella, nei limiti che sono possibili ad una rivista indipendente e priva di finanziamenti, di dare avvio ad un nuovo tipo di comunicazione diretta fra le "periferie" storiografiche. I lettori di Diacronie troveranno a disposizione nei numeri miscellanei alcuni tra i saggi più interessanti di alcuni autori di rilievo facenti parte della comunità scientifica internazionale, originariamente pubblicati nella loro lingua madre e da noi tradotti in lingua italiana. Questo lavoro di traduzione attualmente si basa sul lavoro volontario dei redattori e dei collaboratori di Diacronie.

In questa prima occasione presentiamo un saggio di Georgios Contogeorgis, già professore e rettore dell'Università Panteion di Atene, direttore di tesi al CNRS e - tra il 1985 e il 1989 - direttore generale e poi presidente del canale greco ERT. Durante la sua lunga carriera accademica ha avuto modo di sviluppare alcune teorie che toccano tanto gli studi di politologia quanto il campo filosofico. Epistemologia della democrazia e modernità. La posta in gioco del superamento dell'llluminismo in Europa, l'articolo di Contogeorgis che Diacronie presenta ai lettori in questo numero tratta proprio del cosmosistema ellenico, inserendolo in una riflessione più ampia sulla democrazia nella contemporaneità. La traduzione, ricca e complessa come la prosa di Contogeorgis, è a cura di Irene Vagionakis.

Il secondo articolo del progetto PICO che presentiamo è un'ampia riflessione di Alberto Vieira sui discorsi a favore o contro l'insularità sviluppatisi nell'ambito del dibattito portoghese, con una particolare attenzione nei confronti del caso maderense. Vieira, oltre a presiedere il CEHA (Centro de Estudos de História do Atlântico), è uno dei maggiori specialisti di studi insulari.

Il suo saggio, Il discorso dell'anti-insularità e il poio maderense come sua negazione, parte da un caso di studio peculiare - quello dell'arcipelago di Madera - per affrontare i temi dell'insularità, della retorica prodotta per giustificare una condizione particolare delle popolazioni isolane e dei discorsi sviluppati, al contrario, per negare questa condizione particolare, fondati sull'idea di continuità territoriale.

Particolarmente ricco e utile è l'apparato di note, che permetterà a chi voglia avvicinarsi a questo campo di studi, di avere a disposizione una guida che illustri il dibattito nei suoi termini generali. 


\section{Recensioni}

Con alcune delle recensioni che presentiamo in questo numero ritorniamo sul tema dello Stato e dell'esercizio della violenza.

Il testo di Valerio Romitelli La felicità dei partigiani e la nostra, recensito da Fausto Pietrancosta, ripercorre le forme di sperimentazione politica messe in atto dalle bande partigiane nel biennio 1943-1945. La narrazione punta così a ricostruire un vissuto emozionale che ha accomunato le storie di questi "pionieri" attivi nella società, ma autonomi e non sempre riconducibili all'esperienza nei partiti.

9 Federica Addis si è occupata di prendere in esame il volume di Alessandro Naccarato, Difendere la democrazia. Il PCI contro la lotta armata. Questo saggio indaga il rapporto tra il Partito Comunista Italiano e la lotta armata di sinistra degli anni Settanta ed Ottanta, analizzando in modo approfondito l'atteggiamento, l'interpretazione e la reazione del PCI al terrorismo "rosso", per lungo tempo, e sorprendentemente, poco presenti nell'agenda degli storici.

Il libro di Giovanni Focardi e Cecilia Nubola Nei tribunali (sui processi agli ex-fascisti nell'Italia del dopoguerra), recensito da Andrea Martini, ritorna sul tema della violenza e sulla giustizia a seguito di un cambio di regime.

Riprendendo alcune delle tematiche affrontate nell'articolo di Rafaella Bettamio, Karl Schurster recensisce il volume di Márcia Tiburi, incentrato sulla permanenza di pratiche autoritarie nel Brasile contemporaneo.

Alessandro Stoppoloni ha analizzato il volume di John Foot incentrato su una tematica della storia italiana che ha conosciuto un ampio risalto anche nella pubblicistica corrente: la nuova psichiatria del gruppo di Franco Basaglia.

Federico Mazzini recensisce una raccolta miscellanea curata da Philippe Poirrier che raccoglie alcune ricerche sulla Prima guerra mondiale nell'ottica della storia culturale. Mairon Escorsi Valério prende invece in esame l'opera di Renilson Rosa Ribeiro dedicata alla legittimazione storiografica dell'impero brasiliano operata nel XIX e XX secolo a partire dall'opera di Francisco Adolfo de Varnhagen.

34 La recensione di Sabrina Bassi sul volume Animerama. Storia del cinema d'animazione giapponese della prof.ssa dell'università Ca' Foscari Maria Roberta Novielli la quale fornisce un racconto coinvolgente ed esaustivo dello sviluppo di uno dei prodotti culturali più grandi e conosciuti attualmente in Occidente del paese del Sol Levante: il cinema di animazione.

\footnotetext{
Buona lettura,

Jacopo Bassi e Fausto Pietrancosta
} 


\section{NOTE}

1. S. 1429 - Disegno di Legge costituzionale "Disposizioni per il superamento del bicameralismo paritario, la riduzione del numero dei parlamentari, il contenimento dei costi di funzionamento delle istituzioni, la soppressione del CNEL e la revisione del Titolo V della parte II della Costituzione", approvato in prima deliberazione - dal Senato nella seduta del 13 ottobre 2015 e dalla Camera nella seduta dell'11 gennaio 2016 e - in seconda deliberazione - dal Senato nella seduta del 20 gennaio 2016 e dalla Camera nella seduta del 12 aprile 2016, pubblicato nella Gazzetta Ufficiale della Repubblica Italiana 15 aprile 2016 n. 88.

2. Si vedano a riguardo gli Atti dell'Assemblea Costituente 2 giugno 1946 - 31 gennaio 1948, pubblicati in CAMERA DEI DEPUTATI, Archivio storico, URL: < http://legislature.camera.it/frameset.asp? content=\%2Faltre\%5Fsezionism\%2F304\%2F8964\%2Fdocumentotesto\%2Easp\%3F >, [consultato il 31 agosto 2016].

\section{AUTORI}

\section{JACOPO BASSI}

Jacopo Bassi ha conseguito la laurea specialistica in Storia d'Europa presso l'Università degli Studi di Bologna. Nel corso dei suoi studi si è occupato di storia della Grecia e dell'Albania in età contemporanea e di storia e istituzioni della Chiesa ortodossa. Ha lavorato per le case editrici Éditions des femmes, Il Mulino e Zanichelli. URL: < http://www.studistorici.com/2009/02/24/jacopo_bassi/ >

\section{FAUSTO PIETRANCOSTA}

Fausto Pietrancosta ha conseguito il titolo di dottore di ricerca in Storia presso l'Università di Bologna con una tesi inerente le relazioni tra istituzioni politiche e intervento pubblico in economia nella prospettiva del coordinamento tra amministrazioni centrali ed ente regionale siciliano. Già dottore magistrale in Storia d'Europa, presso la stessa Università con una tesi in Storia dello Stato italiano sul coordinamento costituzionale e l'avvio dell'autonomia regionale siciliana, ha svolto attività di ricerca presso l'Archivio Storico e la biblioteca dell'Istituto Luigi Sturzo di Roma, presso l'Assemblea regionale siciliana, proseguendo poi l'attività di ricerca presso gli archivi degli enti pubblici economici in Sicilia e presso la biblioteca SVIMEZ di Roma. I suoi interessi sono rivolti allo studio dell'evoluzione storica delle autonomie regionali nell'Italia del secondo dopoguerra e delle politiche di intervento a favore dello sviluppo industriale nel Mezzogiorno.

URL: < http://www.studistorici.com/2008/09/14/fausto-pietrancosta/ > 